HIV-infected Egyptians unrelated to the 1993 epidemic were only $76 \%$ to $89 \%$ related to those from outbreak strains.

Based on the remarkable nucleotide sequence homology of the outbreak HIV strains analyzed, it was concluded that HIV infection was introduced into PCA and later into UCA from a common source late in 1992 or early in 1993. The authors pointed out that dialysis patients may be at risk for HIV infection if basic infection control guidelines are not followed.

From: El Sayed NM, Gomatos PJ, Beck-Sague CM, Dietrich U, von Briesen $\mathrm{H}$, Osmanov S, et al. Epidemic transmission of human immunodeficiency virus in renal dialysis centers in Egypt. J Infect Dis 2000;181:91-97.

\section{EPA Issues Stop Sale on Disinfectant Product}

On December 23, 1999, the EPA issued a Stop Sale, Use, or Removal Order for a commercial disinfectant spray because of potential bacterial contamination in hospital and healthcare settings. The order halts sale by the manufacturer of Medaphene Plus Disinfectant Deodorant (EPA registration no. 11694-98) registered by ITW Dymon, Inc (Olathe, KS), until the agency can further evaluate the extent of the contamintaion. The Agency is working with the $\mathrm{CDC}$ to take appropriate steps to notify healthcare facilities nationwide of the order.

The product is currently used on environmental surfaces such as countertops, walls, chart racks, bedside furniture, and wheelchairs in hospitals, medical and dental offices, schools, and other healthcare facilities. It is not used on critical medical devices.

During testing, agency staff determined that three lots of the samples were contaminated with the microorganism Bacillus subtilis, a bacterium that may cause infections in immunosuppressed or traumatized patients. At this time, neither the agency nor the CDC have received any reports regarding incidents linking $B$ subtilis infections to this susceptible group.

For further information, call Ellen Kramer, 202-260-4376.

FROM: EPA issues stop sale on disinfectant product [press release]. Washington, DC: Environmental Protection Agency; December 23, 1999.

\section{Gender-Based Differences in Sepsis Outcomes}

It has been hypothesized that among factors postulated to affect outcome in sepsis is the gender of the patient, with a suggestion that females may have lower mortality. Eachempati and coinvestigators from Department of Surgery, Weill Medical College of Cornell University, New York, conducted a study to test the hypothesis that female patients admitted to the surgical ICU (SICU) with a documented infection have a lower mortality rate. They performed retrospective analysis of a prospectively collected data set in an SICU of a university hospital medical center. They analyzed a consec- utive series of 1,348 patients who had signs of systemic inflammatory response syndrome on admission to an SICU. A cohort of 443 patients (32.9\%) admitted with documented infection-and who therefore had sepsis, severe sepsis, or septic shock-constituted the study population. For each patient, APACHE II and III scores, systemic inflammatory response syndrome score, gender, age, and hospital mortality were recorded. Chi-squared with Fisher's Exact Test was performed to compare mortality rates between males and females. Univariate analysis of variance was used to compare continuous variables in discrete populations. Multivariate analysis of variance (ANOVA) was used to determine which factors independently predicted mortality. Primary outcome measures were mortality, ICU length of stay, hospital length of stay, and maximal multiple organ dysfunction score. Outcomes were stratified by gender.

Patients had a mean age of 67 years and mean APACHE II and III scores of 20.1 and 67.7, respectively. There were no demographic differences between genders. Overall, 104 (23.5\%) of 443 patients with sepsis died. The difference in mortality rates between female and male patients was not significant, except in octogenarians $(P=.05)$. Multivariate ANOVA, APACHE III $(P<.001)$, maximal multiple organ dysfunction score $(P<.001)$, and female gender $(P=.02)$ predicted mortality. In females, APACHE III $(P=.03)$ and maximal multiple organ dysfunction score $(P<.001)$ predicted mortality, but age did not.

It was concluded that female gender is an independent predictor of increased mortality in critically ill surgical patients with documented infection.

FROM: Eachempati SR, Hydo L, Barie PS. Genderbased differences in outcome in patients with sepsis. Arch Surg 1999;134:1342-1347.

\section{Influenza Vaccination of HCWs Reduces Risk to LTCF Elderly}

Vaccination of healthcare workers (HCWs) has been recommended to prevent nosocomial influenza infection of elderly patients in long-term care (LTC). Data are, however, limited on this strategy. Carman and colleagues from the Institute of Virology at the University of Glasgow, United Kingdom, conducted a study to determine whether vaccination of $\mathrm{HCWs}$ lowers mortality and the frequency of virologically proven nosocomial influenza in elderly patients in LTC facilities.

HCWs in 20 long-term elderly care hospitals (range, 44105 patients) were randomly offered or not offered influenza vaccine. All deaths among patients were recorded over 6 months in the winter of 1996-1997. A random sample of 50\% of patients was selected for virological surveillance for influenza, with combined nasal and throat swabs taken every 2 weeks during the epidemic period. Swabs were tested by tissue culture and PCR for influenza viruses $A$ and $B$.

Influenza vaccine uptake in HCWs was $50.9 \%$ in hospitals in which they were routinely offered vaccine, compared with $4.9 \%$ in those in which they were not. The uncorrected rate of mortality in patients was $102(13.6 \%)$ of 749 in vaccine 
hospitals compared with $154(22.4 \%)$ of 688 in no-vaccine hospitals. The two groups did not differ for proportions of patients positive for influenza infection $(5.4 \%$ and $6.7 \%$, respectively); at necropsy, PCR was positive in 0 of 17 patients from vaccine hospitals and $6(20 \%)$ of 30 from no-vaccine hospitals.

The authors concluded that vaccination of HCWs was associated with a substantial decrease in mortality among patients. However, virological surveillance showed no associated decrease in non-fatal influenza infection in patients.

FROM: Carman WF, Elder AG, Wallace LA, McAulay K, Walker A, Murray GD, et al. Effects of influenza vaccination of healthcare workers on mortality of elderly people in long term care: a randomized controlled trial. Lancet 2000;355:93-97.

\section{Surveillance Methods in Australian Hospitals}

The prevalence of nosocomial infection in Australian hospitals is estimated to be between $5.5 \%$ and $6.3 \%$. Since 1989, infection control professionals (ICPs) in hospitals accredited by the Australian Council on Health Care Standards (ACHS) have been encouraged to collect nosocomial infection data according to ACHS methodology. Murphly and McLaws report on the development of methods to perform surveillance. In 1996, they surveyed members of the Australian Infection Control Association to examine the time spent on surveillance, the practice of surveillance of all hospital infections (hospitalwide surveillance), case-finding methods, case definitions, and reporting routinely used by ICPs in acute-care hospitals. They also examined the ICPs' education and experience in infection control (IC).

The survey was completed and returned by $65 \%$ (644/993) of Australian Infection Control Association members. Of the ICPs who completed the survey, $47.8 \%(308 / 644)$ met the criteria for inclusion, because they coordinated an IC program in an acute-care or surgical hospital and performed surveillance for either surgical wound infection, intravascular device-related bacteremia, or non-device-related bacteremia. Of the ICPs who reported their facility's accreditation status, 93.5\% participated in ACHS system. Most (97.6\%) ICPs had completed hospital-based general registered nurse training. Only $1.9 \%(6 / 308)$ of ICPs reported completion of continuing education relating to hospital epidemiology. The number of years of IC experience ranged from 0 to 35 years, with a median of 4 years. ICPs spent a substantial proportion of their total weekly IC time on surveillance irrespective of ACHS accreditation; 19.5 hours in ACHS hospitals and 15.6 hours in nonACHS hospitals $(P=.33)$. More than three quarters $(76.0 \%)$ of ICPs performed hospitalwide surveillance. The case-finding methods, definitions of infections, and reporting formats varied greatly. The definition most commonly applied by ICPs to define surgical wound infection was infection within 30 days after the operative procedure, plus purulent drainage, plus isolation of organisms from a culture from the incision site, plus diagnosis by a medical officer. A five-item definition of a patient being asymptomatic, plus afebrile on admission, plus infection occurring at least 48 hours after admission, plus the patient having a fever of $>38^{\circ} \mathrm{C}$, plus a recognized culture from one or more bottles was used by $15.7 \%$ of ICPs to define a case of bacteremia.

It was concluded that surveillance is the core business of Australian ICPs and consumes a substantial proportion of their time. The importance of surveillance, the epidemiological limitations of the current ACHS system, and the nonstandard methods reported indicates that improved methodology is required for case finding and reporting of nosocomial infections. It was recommended that Australian ICPs should complete training in the principles of surveillance and epidemiology. With this training, ICPs can work collaboratively with other healthcare professionals to develop epidemiologically sound, local, nosocomial surveillance systems and lobby for a voluntary, national, standardized, risk-adjusted system of targeted nosocomial surveillance.

FROM: Murphy CL, McLaws M. Methodologies used in surveillance of surgical wound infections and bacteremia in Australian hospitals. Am J Infect Control 1999;27:474-481.

\section{Pediatric AIDS in Europe and US}

AIDS has shown dramatic increases among women in the last decade, facilitating the spread of pediatric AIDS. Incidence rates of AIDS in children are essential for international comparisons at a population level. Dal Maso and colleagues from the Servizio di Epidemiologia, Centro di Riferimento Oncologico, Aviano, Italy, reported on a study of incidence rates of AIDS 1985 to 1996 by 3-year period in children $<13$ years of age for 15 European countries and, for comparative purposes, the United States. For each country, the rates were adjusted for reporting delay and standardized on the world standard population.

In European countries with $>100$ cases, the steepest rises were seen in the United Kingdom (whose rates increased from $0.8 / 1,000,000$ children in $1985-1987$ to $4.1 / 1,000,000$ in 1994-1996) and Spain (from 4.4/1,000,000 to $16.5 / 1,000,000)$. In 1985 to 1987 , rates were substantially higher in the United States $(8.3 / 1,000,000)$ than in any European country, but in recent years, the rates have become similar to those of Spain. Very elevated rates were observed in Romania, with incidences of $120.4 / 1,000,000$ in the early $1990 \mathrm{~s}$, due to nosocomial HIV transmission. In most countries, 1994 to 1996 rates tended to level off or decrease. In a few countries (Germany, Greece, Denmark, Austria, and The Netherlands), AIDS incidence rates were $<2$ cases per $1,000,000$ children in 1994 to 1996 . The proportion of cases acquired by transfusion, in 1985 to 1996 , is $\sim 20 \%$ or less of pediatric AIDS cases in most countries. No substantial heterogeneity emerges between countries in the distribution of AIDS indicator diseases. The authors concluded that, during 1985 to 1993, pediatric AIDS incidence rates increased in most European countries and leveled off thereafter. No pediatric AIDS epidemic occurred. In a few countries, incidence rates persisted at $\sim 1$ case per $1,000,000$.

FROM: Dal Maso L, Parazzini F, Lo Re A, Favero A, La Vecchia C, Franceschi S. Paediatric AIDS incidence in Europe and the USA, 1985-96. J Epidemiol Biostat 1999;4:75-81. 